\title{
SAÚDE BUCAL E QUALIDADE DE VIDA DOS IDOSOS.
}

\section{ORAL HEALTH AND QUALITY OF LIFE IN THE ELDERLY.}

\author{
Gabriela Furst Vaccarezza* \\ Raissa Lopes Fuga** \\ Stephanie Regiane Prata Ferreira**
}

\begin{abstract}
RESUMO:
Introdução: A população brasileira vem envelhecendo de forma rápida desde o início da década de 60, quando a estrutura etária da população começou a ser alterada. Em 1996 apenas 5\% da população tinham 60 anos ou mais, enquanto que em 2020 é estimado que 9\% da população serão de idosos. (Silva et al., 2000). O objetivo deste estudo é avaliar condições de saúde bucal dos idosos que residem na Vila dos Idosos, coletando informações sobre sua condição de vida, e tentando correlacionar o quanto a saúde bucal pode comprometer a qualidade de vida e autonomia desses idosos. Métodos: A coleta de dados foi ralizada por meio de questionários e exame clínico; foram entrevistados idosos acima de 60 anos, de ambos os sexos. Todas as entrevistas foram realizadas na própria Vila dos Idosos. Resultados: Os resultados mostram que a perda dentária ou o uso de próteses inadequadas implicam impactos negativos na qualidade de vida, especialmente no que se refere à preocupação, estresse decorrente de problemas na boca e à vergonha. Obteve-se na pesquisa que, de maneira geral, a saúde bucal em idosos encontra-se frágil, necessitando de maior atenção dos profissionais da área de saúde; entretanto, quando é analisada a autopercepção de saúde bucal a grande maioria avalia como muito boa ou boa. Conclusão: $\mathrm{O}$ achado mais importante do estudo foi que, apesar dos dados obtidos indicarem uma saúde bucal frágil que revela um comprometimento com a qualidade de vida, a grande maioria dos idosos avaliou positivamente a própria saúde. Isso talvez possa indicar que, para essa população, a saúde bucal está dissociada da saúde.
\end{abstract}

DESCRITORES: Saúde bucal • Qualidade de vida • Perfil de impacto da doença • Saúde do idoso

\section{ABSTRACT :}

Introduction: The Brazilian population is aging rapidly since the early '60s, when the age structure of population began to change. In 1996 only 5\% of the population had 60 years or more, whereas in 2020 it is estimated that $9 \%$ of the population will be elderly. (Silva et al., 2000). The aim of this study is to assess oral health status of the elderly who reside in the "Vila dos Idosos", collecting information about the condition of their life, and trying to correlate if the oral status affetcts the quality of life and autonomy of the elder people. Methods: Through questionnaires, a search was conducted with elderly aged 60-90 years of age, both sexes to determine how oral health affects quality of life. Results: The results show that tooth loss or the use of inadequate dentures involves negative impact on quality of life, especially with regard to the concern, stress arising from problems in the mouth and shame. Was obtained in the research, that in general, oral health in the elderly, is fragile, requiring greater attention to both health professionals, but also to public health. Conclusions: Concluding that through the data, they are indicating a commitment to quality of life.

DESCRIPTORS: Oral health • Quality of life • Sickness impact profile • Health of the elderly 


\section{N T RO DUÇÃ O}

No Brasil, assim como nos países mais desenvolvidos, os progressos tecnológicos e os avanços dos estudos no campo da saúde levam a um aumento na expectativa de vida do homem. (Kina et al. ${ }^{1}$ 1996).

A população brasileira vem envelhecendo de forma rápida desde o início da década de 60, quando a queda das taxas de fecundidade começou a alterar a estrutura etária da população. Em 2020, cerca de $9 \%$ da população terão 60 anos ou mais, contra 5\% em 1996 (Silva e Valsecki Júnior $\left.{ }^{1}, 2000\right)$; (Ramos et al. ${ }^{3}$ 1987)

A saúde bucal tem sido relegada ao esquecimento, no caso brasileiro, quando se discutem as condições de saúde da população idosa. (Colussi e Freitas ${ }^{4}$ 2002)

A perda total de dentes é aceita pela sociedade em geral como algo normal e natural com o avanço da idade, o que é falso. (Rosa et al. ${ }^{5}$ 1992).

No Levantamento Epidemiológico em Saúde Bucal, realizado pelo Ministério da Saúde (MS) em 1986, foi encontrado como resultado a inexistência de medidas eficazes que impeçam a recidiva da cárie na população, fazendo com que haja sempre o surgimento de novas necessidades, que nunca se esgotarão enquanto for mantido o modelo atual de atenção à doença (Fernandes et al. ${ }^{6}$ 1997).

Considerando que nas últimas décadas a saúde bucal não tem sido valorizada pelas pessoas em geral e que a prevenção em Odontologia no Brasil só passou a despertar interesse a partir do final da década de 70 , supõe-se que o grupo populacional de terceira idade deve apresentar baixos níveis de saúde bucal. (Rosa et al. ${ }^{5}$ 1992).

Apesar de não existirem doenças bucais relacionadas diretamente à velhice, alguns problemas, como a diminuição da capacidade mastigatória, a dificuldade de deglutição, a secura na boca, as modificações no paladar e a perda de dimensão vertical têm efeitos cumulativos negativos e prejudiciais para o indivíduo. (Silva e Valsecki Júnior $\left.{ }^{1}, 2000\right)$. Isso faz com o que os idosos tenham dificultade, ou seja, incapazes de desempenhar as atividades cotidianas de forma independente, comprometendo de forma significativa a qualidade de vida. (Brasil'7, 2006).
É através dos programas de promoção de saúde e prevenção de moléstias que se pode dar qualidade na vida a essas pessoas. A qualidade de vida e a falta de autonomia também esta relacionada com a condição bucal.

\section{OB JE T I V 0}

Correlacionar a saúde bucal dos idosos com a qualidade de vida.

\section{MATERIAIS E METÓDOS}

O estudo foi realizado em um grupo de 91 idosos com faixa etária de 60 a 90 anos, de ambos os sexos, que residem na Vila dos Idosos.

Foram aplicados dois questionários, na própria Vila dos Idosos, em espaços isolados, que permitiram atendimento individualizado. Foi utilizado o Oral Health Impact Profile - OHIP- 14, versão em português, e questionário de autoavaliação da saúde bucal, adaptado por Silva e colaboradores. (Silva $\left.{ }^{8}, 1998\right)$

O questionário OHIP-14 vem sendo muito utilizado para indicar os aspectos da qualidade de vida mais afetados pelo estado de saúde bucal e é um forte aliado no estabelecimento de melhores abordagens para atendimento integral ao paciente. Esse instrumento permite indicar as dimensões da qualidade de vida afetadas pela condição de saúde bucal.

O preenchimento do questionário permitiu a coleta de dados para a amostra, incluindo as variáveis de gênero, idade, situação civil, tempo e uso de prótese.

Já o questionário de autoavaliação da saúde bucal, adaptado por Silva e Valsecki Júnior ${ }^{1}, 2000$, tem como finalidade a pesquisa de como os idosos analisam sua saúde bucal, sua autopercepção da saúde.

\section{RESULTADOS}

Foram avaliados 91 idosos com idades variando de 60 a 80 anos, de ambos os sexos, todos com renda de até três salários mínimos. Do total, 43\% eram homens e $57 \%$ mulheres

O OHIP mostrou que $11 \%$ dos idosos avaliaram sua saúde bucal como péssima, $5,5 \%$ como ruim, $27,5 \%$ como regular, $40,7 \%$ como boa e $15,4 \%$ como excelente.
VACCAREZZA GF

FUGA RL

FERREIRA SRP

SAÚdE BUCAL

E QUALIDADE

DE VIDA DOS

IDOSOS.

$\therefore 135$

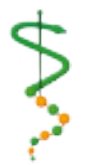

Revista de ODONTOLOGIA DA Universidade Cidade de São Paulo

2010; 22(2):

$134-7$, MAI-AGO 
VACCAREZZA GF

FUGA RL

FERREIRA SRP

SAÚDE BUCAL

E QUALIDADE

$D E \quad V I D A \quad D O S$

IDOSOS.

\section{6}

REVISTA DE ODONTOLOGIA DA: UNIVERSIDADE CIDADE DE SÃO PAULO: $2010 ; 22(2):$ $134-7$, MAI-AGO
Em relação à autopercepção da saúde bucal, $10 \%$ dos idosos avaliaram sua saúde bucal como péssima; 5\% como ruim; $29 \%$ como regular; $42 \%$ como boa e $14 \%$ como ótima.

Em relação aos problemas com os seus dentes, $34,1 \%$ relatam ter ou estar com problemas nos dentes, $24,2 \%$ negam qualquer tipo de adversidade, e 65,9\% admitem ter problemas na gengiva.

Foi observado a presença de hiperplasia fibrosa inflamatória em região de fundo de sulco e palato, estomatite protética, língua saburrosa, queilite angular e cálculo salivar. Foram examinadas hiperplasia fibrosa inflamatória em região de fundo de sulco e palato, estomatite protética, língua saburrosa, queilite angular e presença de cálculo salivar.

Também foram encontradas alterações bucais dentro das normalidades, como as varicosidades linguais e manchas melânicas.

Outro fator importante a ser relatado nesta pesquisa foi a porcentagem alta de idosos que utilizam próteses totais: 93\% dos pesquisados são pacientes portadores de próteses superiores e ou inferiores.

O fator emocional desses idosos também foi avaliado e demonstraram a não aceitação das perdas dos dentes, seus sentimentos com a perda dentária são negativos.

Foi notado um grande comprometimento funcional como comer, mastigar ou falar; e social como mudanças no comportamento, insatisfação com a aparência, prejuízo na aceitação social.

Relatos dos idosos mostram que a ausência de dentes dificulta mastigar alimentos duros e consistentes. Relatam vergonha pela ausência dos dentes com consequente obstáculo para a fala.

\section{DISCUSSÃO E CONCLUSÃO}

Observa-se que a sua condição clínica, de maneira geral, é precária. Os dados encontrados sugerem que a qualidade de vida de grande parte dos participantes do estudo estava comprometida, de alguma maneira, pelas condições bucais e/ou suas sequelas. Apesar disso, mais da metade dos idosos avaliados considera que a saúde bucal estava boa ou excelente. Esse fato pode demonstrar que, de alguma forma, esses idosos não levam em consideração a saúde bucal quando pensam em sua vida e na qualidade desta. Como se a saúde bucal estivesse separada da saúde.

Após análise dos dados, ficou evidente que a maioria das pessoas vê sua condição bucal de maneira favorável, mesmo em condições clínicas não satisfatórias. Tal fato pode ser explicado porque as medidas clínicas de saúde utilizadas pelos profissionais preceptores são relativamente fracas quanto à percepção de saúde bucal das pessoas.

Estes resultados apontam uma maior necessidade dos idosos à atenção aos serviços públicos de saúde. Além da implantação de atendimento curativo e reabilitador, seria também necessário o desenvolvimento de ações preventivas e educativas. (Silva e Valsecki Júnior ${ }^{1}$, 2000)

Lamentavelmente, os objetivos da prótese que motivam seu emprego na reabilitação do paciente, visando estética, função, fonética e conforto, podem, devido à instalação de próteses mal adaptadas e à falta de orientação do paciente, afetar de forma adversa o prognóstico final do tratamento, com o aparecimento, por exemplo, de lesões orais (Goiato et al. ${ }^{9}, 2005$ ).

O comprometimento funcional e emocional não pode ser esquecido pelos profissionais ao cuidar dos idosos.

Apesar de ser um estudo exploratório, com poucos casos, outra questão se mostra importante: a implementação de um serviço de prótese dentária no setor público. Tal medida deveria ser encarada como profilática, uma vez que a falta de dentes acarreta outros problemas de saúde, agravando os já existentes e piorando a qualidade de vida da população idosa brasileira (Colussi e Freitas ${ }^{4}$, 2002). 
1. Silva SRC, Valsecki Júnior A. Avaliação das condições de saúde bucal dos idosos em um município brasileiro. Rev Panam Salud Publica/Pan Am J Public Health 2000; 8(4): 268-71.

2. Kina S, Conrado CA, Brenner AJ, Kurihara E. O ensino da estomatogeriatria no Brasil: a experiência de Maringá. Rev Odontol Univ São Paulo 1996 jan-mar; 10(1): 69-73.

3. Ramos LR, Veras RP, Kalache A., "Envelhecimento populacional: uma realidade brasileira". Rev. Saúde Pública., São Paulo, 1987 jun; 21 (3): 211-24.

4. Colussi CF, Feitas SFT. Aspectos Epidemiológicos da saúde bucal do idoso no Brasil. Cad Saúde Publica, 2002 set-out; 18(5): 1313-20.

5. Rosa AGF, Fernandez RA, Pinto VG, Ramos LR. Condições de saúde bucal em pessoas de 60 anos ou mais no município de São Paulo (Brasil). Rev Saúde Pública, 1992 jun; 26(3): 155-60.

6. Fernandes RAC, Silva SRC, Watanabe MGC, Pereira AC, Martildes MLR. Uso e necessidade de prótese dentária em idosos que demandam um Centro de Saúde. Rev Bras Odontol 1997 mar-abr; 54(2): 107-10.

7. Brasil:Ministério da Saúde: Cadernos de atenção básica: envelhecimento e saúde da pessoa idosa. Brasília: Ministério da Saúde; 2006

8. Silva SRC. Autopercepção das condições bucais em pessoas com 60 anos e mais de idade. [Tese] São Paulo: Faculdade de Saúde Pública, USP; 1998.

9. Goiato MC, Casteleoni L, Santos DM, Genari Filho H, Assunção WG. Lesões orais provocadas pelo uso de próteses. Pesq Bras Odontopediatria Clin Integr 2005 janabr; 5(1): 85-90.

Recebido em: 08/06/2010.

Aceito em: 16/03/2010.

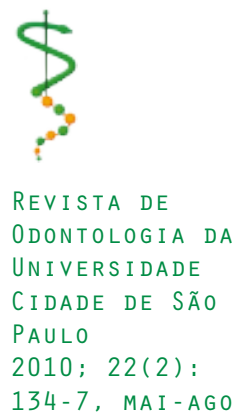

